\title{
A phase I trial of ANG1/2-Tie2 inhibitor trebaninib (AMG386) and temsirolimus in advanced solid tumors (PJC008/NCI\#9041)
}

\author{
Joanne W. Chiu ${ }^{1,2}$ • Sebastien J. Hotte ${ }^{3}$ Christian K. Kollmannsberger ${ }^{4}$. \\ Daniel J. Renouf ${ }^{4}$ - David W. Cescon ${ }^{1,2}$ - David Hedley ${ }^{1,2}$ - Sue Chow ${ }^{1,2}$. \\ Jeffrey Moscow $^{5}$ - Zhuo Chen ${ }^{1,2}$ - Meghan Perry ${ }^{1,2}$ - Ivan Diaz-Padilla ${ }^{1,2}$ - David Tan ${ }^{1,2}$. \\ Hal Hirte $^{3}$ - Elaine McWhirter ${ }^{3}$ Helen Chen ${ }^{5}$ Lillian L. Siu ${ }^{1,2}$ - Philippe L. Bedard ${ }^{1,2}$
}

Received: 14 October 2015 / Accepted: 3 December 2015 /Published online: 19 December 2015

(C) The Author(s) 2015. This article is published with open access at Springerlink.com

Summary Background There is crosstalk between the ANGTie2 and the PI3K/Akt/mTOR pathways. Combined ANG1/2 and mTOR blockade may have additive anti-cancer activity. The combination of trebananib, an inhibitor of ANG1/2-Tie2 interaction, with temsirolimus was evaluated in patients with advanced solid tumors to determine tolerability, maximum tolerated dose (MTD), and preliminary antitumor activity. Methods Patients were enrolled using $3+3$ design, and were given intravenous trebananib and temsirolimus on Day 1,8, 15 and 22 of a 28-day cycle. Dose limiting toxicities (DLTs) were evaluated during cycle 1 . Peripheral blood was collected for evaluation of Tie2-expressing monocytes (TEMs) and thymidine phosphorylase (TP). Sparse pharmacokinetic (PK) sampling for trebananib drug levels was performed on Day 1 and 8 of cycle 2. Results Twenty-one patients were enrolled, 6 at dose level (DL) 1, 7 at DL -1 , and 8 at DL -2 . No effect of temsirolimus on trebananib PK was observed. The most

Electronic supplementary material The online version of this article (doi:10.1007/s10637-015-0313-8) contains supplementary material, which is available to authorized users.

Philippe L. Bedard

philippe.bedard@uhn.ca

1 Division of Medical Oncology \& Hematology, Princess Margaret Cancer Centre, University Health Network, Suite 5-125, 610 University Avenue, Toronto, ON M5G 2M9, Canada

2 Department of Medicine, University of Toronto, Toronto, ON, Canada

3 Juravinski Cancer Centre and Escarpment Cancer Research Institute, Hamilton, ON, Canada

4 British Columbia Cancer Agency, Vancouver, BC, Canada

5 National Cancer Institute, Cancer Therapy Evaluation Program, Rockville, MD, USA common treatment-related adverse events (AEs) were: fatigue ( $81 \%$ ), edema (62\%), anorexia (57\%), nausea (52\%), rash $(43 \%)$ and mucositis $(43 \%)$. The most common grade $\geq 3$ AEs included lymphopenia (28\%) and fatigue (28\%). The MTD was exceeded at DL-2. Of 18 response evaluable patients, 1 partial response was observed (ER+/HER2-/PIK3CA mutant breast cancer) and 4 patients had prolonged $\mathrm{SD} \geq 24$ weeks. No correlation with clinical benefit was observed with change in number TEMs or TP expression in TEMs with treatment. Conclusions The MTD was exceeded at trebananib $10 \mathrm{mg} / \mathrm{kg}$ weekly and temsirolimus $20 \mathrm{mg}$ weekly, with frequent overlapping toxicities including fatigue, edema, and anorexia.

Keywords AMG386 - Trebananib - Temsirolimus · Phase 1 . Combination therapy

\section{Introduction}

Approved agents against the vascular endothelial growth factor (VEGF) pathway and its receptor (VEGFR), such as bevacizumab, aflibercept, and sunitinib, provide proof of principle of targeting angiogenesis. However, the efficacy of these anti-VEGF therapies is suboptimal and the duration of response is often short-lived. It has been postulated cancer may not respond to anti-VEGF therapy or regain growth potential through the development of evasive resistance. Among the proposed mechanisms include up-regulation of alternative pro-angiogenic signals, protection of tumor vasculature by recruitment of pro-angiogenic inflammatory cells or by increasing protective pericyte coverage [1].

Inhibition of the angiopoietin-Tie2 system presents an attractive strategy to circumvent resistance to anti-VEGF therapy. Angiopoietins (ANG) are a family of secreted ligands of 
the endothelial receptor Tie2 that play a critical role in initiation of tumor angiogenesis, tumor inflammation and metastasis [2]. There are 3 members of ANG known to participate in angiogenesis in human. ANG-1 has both pro- and antiangiogenic functions, but the exact role has not been well characterized [3-6]. ANG-2 is believed to be the primary ANG involved in angiogenesis in malignant setting. It is frequently up-regulated at areas of angiogenesis within tumors. Elevated expression of this ligand is associated with more advanced disease and poor prognosis [7-10]. The function of ANG-4 is unclear, as it has a restricted pattern of expression in lung [11].

Trebananib (AMG386) is a first-in-class selective antagonist peptide-Fc fusion protein inhibiting the interaction between ANG-1, ANG-2 and Tie2. In xenograft models, this agent demonstrated potent anti-tumor activity, accompanied by suppressed endothelial cell proliferation [7]. The phase 1 clinical trial of trebananib in patients with advanced solid tumors showed favorable tolerability and preliminary antitumor activity [12]. The most common toxicities were fatigue and peripheral edema; few patients experienced treatmentrelated toxicities greater than grade 1 . The maximumtolerated dose (MTD) was not reached. One patient with refractory ovarian cancer achieved partial response associated with prolonged treatment of 156 weeks. Trebananib given at $10 \mathrm{mg} / \mathrm{kg}$ intravenously (IV) weekly can be safely combined with several standard chemotherapy regimens [13].

In view of its favorable safety profile, it was hypothesized that trebananib may be suitable for combination therapy with other anti-angiogenic agents. The mammalian target of rapamycin (mTOR) is a protein kinase of the PI3K/Akt signaling pathway with known critical role in cancer growth and tumor angiogenesis [14]. There is crosstalk between the ANGTie2 and the PI3K/Akt/mTOR pathways, as activation of Tie2 receptor leads to recruitment of the p85 regulatory subunit of PI3K and downstream activation of Akt [15]. ANG-1 modulates the survival, migration and sprouting of endothelial cells through PI3K/Akt activation. Temsirolimus is a mTORC1 inhibitor approved for the treatment of advanced renal cell carcinoma (RCC) and mantle-cell lymphoma [16, 17]. Here, we report the results of a phase $1 \mathrm{~b}$ trial of trebananib in combination with temsirolimus in patients with advanced solid tumors.

\section{Patients and methods}

\section{Study objectives}

The primary objectives of this phase $1 \mathrm{~b}$ trial were to evaluate the safety and tolerability of escalating doses of trebananib in combination with temsirolimus, to explore the dose-limiting toxicities (DLTs), and to determine the recommended phase 2 dose (RP2D). The secondary objectives were to evaluate preliminary tumor response in patients with advanced solid tumors and to assess the pharmacodynamics effects with the goal of identifying potential predictive markers that warrant further exploration.

\section{Eligibility criteria}

Inclusion criteria included age of 18 years or older with histologically or cytologically confirmed advanced solid tumors, and for which standard anticancer treatment did not exist or were no longer effective; Eastern Cooperative Oncology Group (ECOG) performance status of $0-1$; life expectancy $\geq 12$ weeks; Response Evaluation Criteria in Solid Tumors (RECIST) v1.1 measurable disease; well-controlled blood pressure; adequate bone marrow, renal, liver functions and lipid profile. Key exclusion criteria included central nervous system metastasis; coagulopathy or history of clinically significant bleeding within 6 months; venous or arterial thromboembolism within 12 months; unresolved toxicities from prior systemic therapy $\geq$ grade 2 by CTCAE version 4.0 ; prior treatment by trebananib or medication targeting ANG or Tie 2 receptor; clinically significant cardiovascular disease within 12 months; major surgery within 28 days; treatment within strong immune modulators within 30 days; non-healing wound, ulcer, or fracture; chemotherapy or radiotherapy within 4 weeks or other investigational drug within 21 days; concomitant administration of strong CYP3A4 inhibitors or inducers. Written informed consent was obtained from all participants included in the study prior to screening procedures. This study was approved by local Institutional Review Boards.

\section{Study design and dose de-escalation}

Dose exploration to identify the MTD followed a $3+3$ design. Trebananib was administered intravenously (IV) over $60 \mathrm{~min}$, followed by temsirolimus IV over 30-60 min on day 1, 8, 15, and 22 of each 28-day cycle. The starting dose level (DL1) of trebananib $15 \mathrm{mg} / \mathrm{kg}$ and temsirolimus at its approved dose of $25 \mathrm{mg}$ was based on the expected tolerability of the combination. Due to the toxicities encountered, reduced dose levels of trebananib $15 \mathrm{mg} / \mathrm{kg}$ and temsirolimus $20 \mathrm{mg}$ weekly (DL-1) and trebananib $10 \mathrm{mg} / \mathrm{kg}$ and temsirolimus $20 \mathrm{mg}$ weekly (DL-2) were explored.

\section{Definition of DLTs and MTD}

Toxicities were graded according to the National Cancer Institute Common Terminology Criteria for Adverse Events (NCI CTCAE) v4.0. DLTs were determined by treatmentrelated toxicities that occurred during cycle 1 . DLT criteria included non-hematologic toxicity that was grade $(G) 4$, or G3 which did not resolve to G1 within $48 \mathrm{~h}$; toxicities that 
resulted in failure to receive $\geq 75 \%$ of the pre-planned doses of trebananib and/or temsirolimus; neutropenia $<0.5 \times 10^{9} / \mathrm{L}$ lasting $\geq 7$ days, neutropenic fever, thrombocytopenia $<25 \times 10^{9} / \mathrm{L}$ or thrombocytopenic bleeding. The MTD was defined as the highest DL at which $\leq 1 / 6$ patients experienced DLTs during first cycle.

\section{Evaluation of safety}

Toxicities were recorded for patients who received one or more doses of trebananib and temsirolimus. Vital signs and weight were measured weekly before each dose of study treatment during cycle 1 and then pre-dose before every subsequent cycle. Complete blood count was assessed before every treatment. Serum chemistry including cholesterol and triglycerides were assessed pre-dose weekly during cycle 1 and the pre-dose day 1 and day 15 of subsequent cycles. Urine protein was measured via dipstick pre-dose day 1 and day 15 of cycle 1 and then pre-dose day 1 of every subsequent cycle. Serum for assessment of anti-trebananib antibodies was collected pre-dose Cycle 1, Day 1, pre-dose Cycle 2, Day 8, and at the end of treatment (EOT) visit approximately 30 days after the last dose of trebananib.

\section{Evaluation of tumor response}

Patients with measurable disease were evaluated for response by imaging or clinical examination every 8 weeks using the Response Evaluation Criteria in Solid Tumors (RECIST) version 1.1 [18]. A confirmatory scan was obtained 4 weeks following initial documentation of an objective response.

\section{Sparse pharmacokinetics (PKs) and anti-trebananib antibodies}

Plasma samples were collected at cycle 2 day (D) 1 (pre-dose and end of infusion at $10 \mathrm{~min}$ ) and cycle $2 \mathrm{D} 8$ (pre-dose) for trebananib concentrations, and at cycle $1 \mathrm{D} 1$ (pre-dose), cycle 2 D8 (pre-dose) and EOT for presence of anti-trebananib antibodies. Both analyses of PKs anti-trebananib antibodies were performed by Amgen using previously published assays [12].

\section{Correlative studies}

Peripheral blood was collected for correlative studies on cycle 1 D1, D3, D8 prior to drug administration (except D3), on cycle $2 \mathrm{D} 1$ prior to and $4 \mathrm{~h}$ after drug administration, and at the EOT visit. Tie2 is expressed by monocytes and the role of Tie-2 expressing monocytes (TEMs) as a potential marker of response was explored. Thymidine phosphorylase (TP) is an angiogenic enzyme, which is increased in TEM upon Tie2 stimulation. Two separate staining procedures were employed: extracellular Tie2 staining with phycoerythrinconjugated anti-Tie 2 antibody after hypotonic red blood cell lysis; intracellular TP staining in a second aliquot of blood followed fixation with formaldehyde and permeabilization with Triton X-100, using indirect TP staining. CD45 and CD33 were used for gating on monocytes and lymphocytes. To normalize results, a ratio of the Median Fluorescence Intensities (MFI) between Monocytes and Lymphocytes (M/L ratio) was calculated for each sample. Associations between baseline Tie2 and TP M/L ratios, and changes in M/L ratios over time with best radiographic response in target lesions were explored. The assay has been verified with blood samples from healthy volunteers [19].

Circulating angiogenic factors (CAFs) including, soluble vascular cell adhesion molecule 1 (sVCAM-1), placental growth factor (PlGF), and VEGF-A were measured at the aforementioned time points using enzyme-linked immunosorbent assay (ELISA) kits from R\&D System (Minneapolis, MN, USA). The assays were performed according to manufacturer's instructions. We hypothesized that these pharmacodynamic markers would rise following the administration of studied drugs. For PIK3CA mutation status, formalin fixed paraffin embedded (FFPE) archival tumor tissue was collected. Molecular characterization was performed using the customized multiplex MassARRAY Sequenom (Sequenom, San Diego, U.S.A.) PMH v1.0 panel or Illumina MiSeq TruSeq Amplicon Cancer Panel (Illumina, San Diego, U.S.A.) in the University Health Network CLIA-certified laboratory. These results were correlated with tumor response.

\section{Statistical analysis}

Descriptive statistics were used to summarize demographic, safety, antitumor activity, and correlative results. Comparison between treatment groups was performed using t-test. Continuous data were presented as mean and standard error; and categorical data were summarized using frequency and percentage. Difference with $p$-value of $<0.05$ is considered statistically significant.

\section{Results}

\section{Patient characteristics}

From April 2012 to March 2014 a total of 21 patients were enrolled. Patient characteristics are summarized in Table 1. The reasons for discontinuation included progressive disease $(n=11)$, toxicity $(n=5)$, withdrawal of consent $(n=3)$, death on study during cycle $1(n=1)$, and development of brain metastasis during cycle $1(n=1)$. All 3 patients who withdrew consent were due to intolerable toxicity, including one patient 
Table 1 Patient characteristics

\begin{tabular}{lll}
\hline & Characteristics & No. $\%$ \\
\hline Gender & Female & $16(76 \%)$ \\
& Male & $5(24 \%)$ \\
Age & Median & 58 \\
& Range & $32-74$ \\
ECOG performance score & 0 & $5(24 \%)$ \\
\multirow{3}{*}{ Primary tumor type } & 1 & $16(76 \%)$ \\
& Ovary & 4 \\
& Colon & 4 \\
& Lung & 2 \\
Prior therapy & Neuroendocrine & 2 \\
Previous chemotherapy regimens & Others & 9 \\
& Chemotherapy & $21(100 \%)$ \\
& 1 & $2(9 \%)$ \\
& 2 & $2(9 \%)$ \\
& 3 & $8(38 \%)$ \\
& $\geq 4$ & $9(43 \%)$ \\
& Median (range) & $3(1-11)$
\end{tabular}

${ }^{a}$ Others: include one of each of breast cancer, gastric cancer, gallbladder cancer, thyroid cancer, bladder cancer, renal cell carcinoma, endometrial mixed carcinoma, bone chordoma, and endometrial sarcoma

who remained on study for 37 weeks with best response of stable disease (SD) before withdrawal.

\section{DLT and MTD}

A summary of DLT events and the dose levels explored are presented in Table 2. In DL 1, 1 of 6 patients experienced DLT with G2 pneumonitis. Due to frequent G1 and 2 AEs including fatigue, nausea, edema, thrombocytopenia, mucositis and rash (Table 3) in DL1, de-escalation to DL-1 was explored. DLTs were experienced by 2 patients, including G3 mucositis and intolerable $\mathrm{G} 2$ limb edema leading to a treatment interruption that did not allow for initiating cycle 2 until more than 14 days after the planned start date. Further de-escalation to DL -2 was explored, and 2 of 6 evaluable patients experienced DLTs, including one episode of G4 hypertriglyceridemia and one death. This patient who died had advanced sigmoid colon cancer and failed 4 lines of systemic therapy before joining the study. On cycle 1 day 7 he presented with persistent diarrhea and was admitted. He then developed fever related to Clostridium Septicum bacteremia. Despite aggressive treatment, he rapidly deteriorated and succumbed to multi-organ failure. A postmortem examination showed that the patient had a transmural recurrence at the site of a prior sigmoid colon anastomosis, with a walled off perforation at this site with necrosis and abscess formation. These AEs were considered to be probably related to study treatment. MTD was exceeded at DL-2 and no further dose de-escalation was explored.

\section{Adverse events possibly related to treatment}

AEs by dose level are listed in Table 3. The most common non-hematologic AEs related to treatment reported in more than $30 \%$ of patients were fatigue ( $81 \%$ ), edema of limbs (62\%), anorexia (57\%), nausea (52\%), rash (43\%), oral mucositis (43\%), increased aspartate aminotransferase (43\%), hyperglycemia (38\%) and diarrhea (33\%). Common hematologic AEs included anemia (43\%), lymphopenia $(57 \%)$ and thrombocytopenia (48 \%). The most frequent $\mathrm{G} \geq 3$ AEs were fatigue ( $28 \%$ ) and lymphopenia (28\%). Five AEs of $\mathrm{G} \geq 4$ occurred: one patient in DL-2 developed G4 hypertriglyceridemia and there was a treatment-related death associated with G4 diarrhea, sepsis and G5 multi-organ failure (details described above). No dose-related trend in the incidence or severity AE was observed. During treatment, 7 patients required dose reduction ( $33 \%$ ) or had 1 of the 2 drugs discontinued due to AEs.

\section{Antitumor activity}

Eighteen of the 21 enrolled patients had at least one postbaseline tumor evaluation for treatment response. Figure 1 summarizes the antitumor response and duration of treatment in each evaluable patient. Six patients (33\%) had progressive disease (PD) as best response. One patient with inflammatory hormone receptor (HR)-positive HER2 negative breast cancer

Table 2 Dose escalation results

\begin{tabular}{llllll}
\hline $\begin{array}{l}\text { Dose } \\
\text { level }\end{array}$ & $\begin{array}{l}\text { Trebananib } \\
(\mathrm{mg} / \mathrm{kg})\end{array}$ & $\begin{array}{l}\text { Temsirolimus } \\
(\mathrm{mg})\end{array}$ & $\begin{array}{l}\text { No. patients treated } \\
(n=21)\end{array}$ & No. DLT & DLT \\
\hline 1 & 15 & 25 & $6(1 \mathrm{IE})$ & 1 & G2 pneumonitis \\
-1 & 15 & 20 & 7 & 2 & $\begin{array}{l}\text { G3 mucositis } \\
\text { G2 intolerable edema } \\
-2\end{array}$ \\
10 & 20 & $8(2 \mathrm{IE})$ & 2 & $\begin{array}{l}\text { G4 sepsis, G4 diarrhea, } \\
\text { G5 multi-organ failure } \\
\text { (same patient) } \\
\text { G4 hypertriglyceridemia }\end{array}$ \\
\hline
\end{tabular}

IE inevaluable 
Table 3 Frequent or significant adverse events (possibly related to treatment)

\begin{tabular}{lllll}
\hline Adverse events & $\begin{array}{l}\text { All grades } \\
\text { No. Patient (\%) }\end{array}$ & $\begin{array}{l}\text { Dose level } \\
(\mathrm{DL} 1 /-1 /-2)\end{array}$ & $\begin{array}{l}\geq \text { Grade } 3 \\
\text { No. Patient }(\%)\end{array}$ & $\begin{array}{l}\text { Dose level } \\
(\mathrm{DL} 1 /-1 /-2)\end{array}$ \\
\hline Fatigue & $17(81 \%)$ & $5 / 7 / 5$ & $6(28 \%)$ & $2 / 1 / 3$ \\
Edema (limbs) & $13(62 \%)$ & $5 / 5 / 3$ & $1(5 \%)$ & $0 / 1 / 0$ \\
Anorexia & $12(57 \%)$ & $3 / 5 / 4$ & $1(5 \%)$ & $0 / 0 / 1$ \\
Lymphopenia & $12(57 \%)$ & $4 / 3 / 5$ & $6(28 \%)$ & $3 / 1 / 2$ \\
Nausea & $11(52 \%)$ & $5 / 2 / 4$ & 0 & - \\
Thrombocytopenia & $10(48 \%)$ & $4 / 2 / 4$ & $1(5 \%)$ & $0 / 0 / 1$ \\
Maculopapular rash & $9(43 \%)$ & $3 / 3 / 3$ & 0 & - \\
Mucositis & $9(43 \%)$ & $3 / 4 / 2$ & $1(5 \%)$ & $0 / 1 / 0$ \\
AST elevation & $9(43 \%)$ & $2 / 5 / 2$ & $3(14 \%)$ & $1 / 1 / 1$ \\
Hyperglycemia & $8(38 \%)$ & $3 / 1 / 4$ & 0 & - \\
Diarrhea & $7(33 \%)$ & $2 / 2 / 3$ & $1(5 \%)$ & $0 / 0 / 1^{\mathrm{a}}$ \\
Hypertension & $7(33 \%)$ & $1 / 4 / 2$ & 0 & - \\
ALT elevation & $6(28 \%)$ & $1 / 2 / 3$ & $2(9 \%)$ & $1 / 0 / 1$ \\
Hypertriglyceridemia & $6(28 \%)$ & $2 / 2 / 2$ & $2(9 \%)^{\mathrm{a}}$ & $1 / 0 / 1^{\mathrm{a}}$ \\
Pleural effusion & $4(19 \%)$ & $2 / 0 / 2$ & $1(5 \%)$ & $0 / 0 / 1$ \\
Pneumonitis & $2(9 \%)$ & $2 / 0 / 0$ & 0 & - \\
Sepsis & $1(5 \%)$ & $0 / 0 / 1$ & $1(5 \%)$ & $0 / 0 / 1^{\mathrm{a}}$ \\
Multi-organ failure & $1(5 \%)$ & $0 / 0 / 1$ & $1(5 \%)$ & $0 / 0 / 1^{\mathrm{b}}$ \\
\hline
\end{tabular}

ALT Alanine aminotransferase, AST Aspartate aminotransferase

${ }^{\text {a }}$ Grade 4

${ }^{\mathrm{b}}$ Grade 5

who had received 11 prior lines of treatment achieved partial response (PR) and remained on study for 51 weeks. Eleven patients $(61 \%)$ had stable disease (SD), in which 4 had prolonged SD for 24 weeks or longer. Tumor type, best response, and progression free survival (PFS) of last line of treatment prior to recruitment, as reference of growth
Fig. 1 Waterfall plot of best treatment response
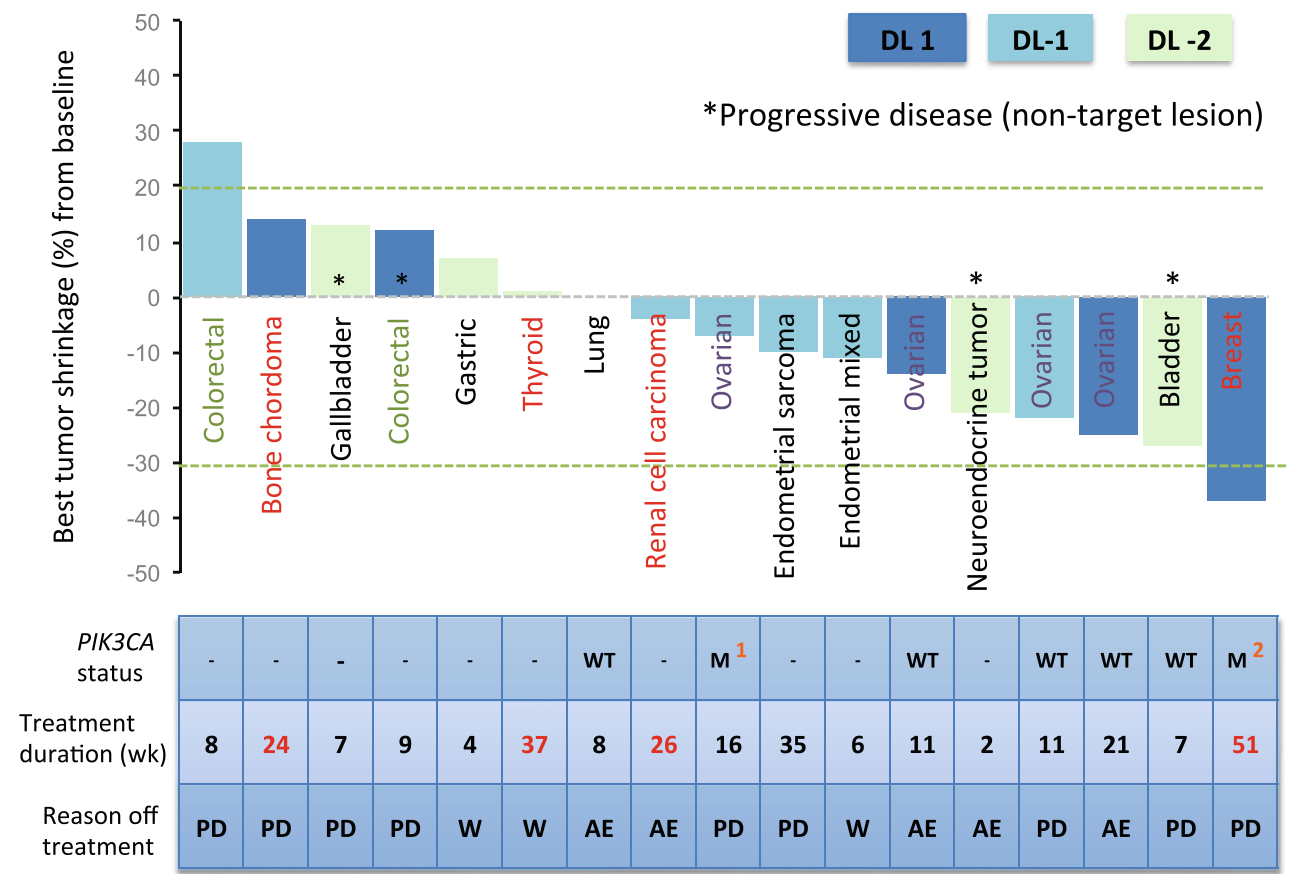

WT: wild type; M: mutation - ${ }^{1} \mathrm{H} 1047 \mathrm{R} ;{ }^{2} \mathrm{~N} 345 \mathrm{~K}$ PD: progressive disease; $A E$ : adverse events; $W$ : withdrew 
modulation for these 4 patients, are as follows: 1 renal cell carcinoma (SD, 7 months), 1 thyroid cancer (PR, 7.5 months), 1 endometrial sarcoma (PD, 1 month), and 1 bone chordoma (PD, 1.5 month). This information suggested that the prolonged disease stabilization observed in the patients with endometrial sarcoma and bone chordoma was probably related to study drugs rather than slow growing biology.

\section{Correlative studies}

\section{PIK3CA mutation status}

Tumor tissue for molecular profiling was available for 8 patients. Two cases with PIK3CA mutation were observed in which 1 patient had best response of PR (breast cancer, PIK3CA mutation at $\mathrm{N} 345 \mathrm{~K}$, treatment duration 51 weeks) and another patient had SD (ovarian cancer, PIK3CA mutation at $\mathrm{H} 1047 \mathrm{R}$, treatment duration 16 weeks). Correlation of PIK3CA mutation with treatment response was not done due to small sample size.

\section{Circulating angiogenic factors (CAFs)}

There was a trend for sustained elevation of sVCAM-1 following treatment but it did not reach statistical significance. The magnitude of SVCAM-1 elevation did not appear to be related to the dose of trebananib or temsirolimus (Fig. 2a). For PlGF, there appeared to be elevation of this CAF with the highest dose of temsirolimus (DL1) (not statistically significant) that was not observed with the 2 lower dose levels (Fig. 2b). The change in VEGF-A over time did not show a specific trend and there was significant overlap in values between different dose levels (Fig. 2c). No correlation between the baseline values of CAFs and duration of treatment was detected (data not shown).

\section{Tie2-expressing monocytes (TEMs) and thymidine phosphorylase (TP)}

Blood for TEMs and TP analysis from the 11 patients treated in Princess Margaret Cancer Centre was available. No discrete population of Tie-2 expressing cells was present in peripheral blood. No association between either baseline TP or Tie2 M/L and radiographic response was observed, and no consistent pattern of change in TP or Tie2 M/L was observed during study therapy (Supplementary figure 3).

\section{Pharmacokinetics (PKs) and anti-trebananib antibodies}

Sparse PK analysis of trebananib taken during cycle 2 is shown in Supplementary figure 4. There was more than 10fold increase in plasma level of trebananib after $10 \mathrm{~min}$ of
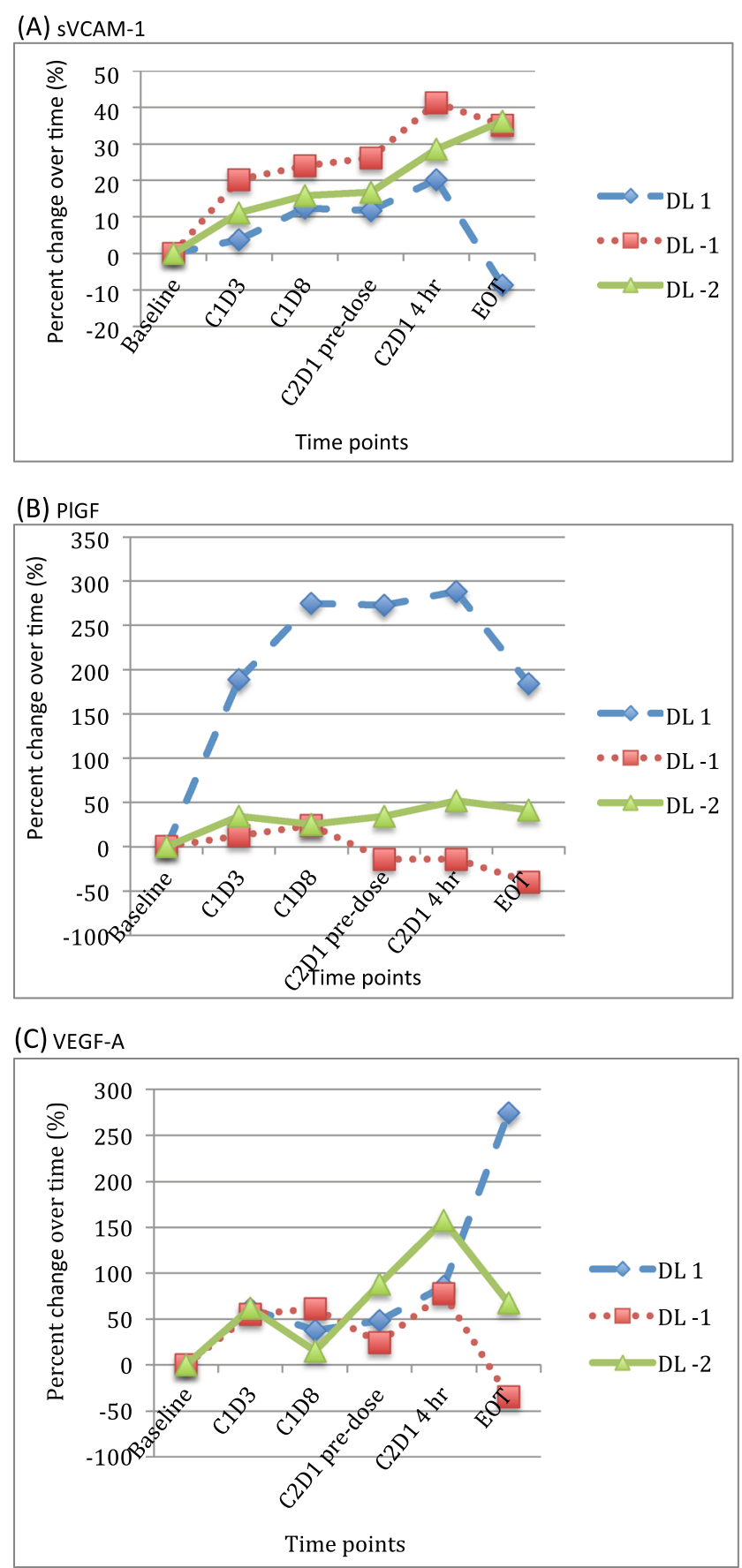

Fig. 2 Average percent change of various circulating angiogenic factors during treatment. a sVCAM-1; b PIGF; c VEGF-A

temsirolimus dosing at all dose levels $(p<0.01)$. The plasma concentration of trebananib returned to trough levels on D8. The post-dose plasma concentration trebananib for DL-1 (temsirolimus $20 \mathrm{mg}$, trebananib $15 \mathrm{mg} / \mathrm{kg}$ ) was significantly higher than DL-2 (temsirolimus $20 \mathrm{mg}$, trebananib $10 \mathrm{mg} / \mathrm{kg}$ ) $(p<0.01)$. Similar trend was observed for DL1 (temsirolimus $25 \mathrm{mg}$, trebananib $15 \mathrm{mg} / \mathrm{kg}$ ) but did not reach significant difference, likely related to an exceptionally lower value for one sample. The post-dose and trough levels of trebananib in 
DL-2 were similar to the first-in-human phase I trial [12]. Similarly, the post-dose and trough levels of trebananib in DL1 and DL-1 with trebananib $15 \mathrm{mg} / \mathrm{kg}$ dosing were between the values at $10 \mathrm{mg} / \mathrm{kg}$ and $30 \mathrm{mg} / \mathrm{kg}$ observe in the phase I trial, suggesting that temsirolimus might not affect the PK of trebananib. No anti-trebananib antibodies were detected during treatment or at end of treatment.

\section{Discussion}

This study explored the feasibility of combining the ANG1/2-Tie2 inhibitor trebananib with temsirolimus. The MTD was exceeded at the lowest dose level of trebananib $10 \mathrm{mg} / \mathrm{kg}$ weekly and temsirolimus $20 \mathrm{mg}$ weekly. No tolerable dosing regimen could be identified for the combination. Frequent overlapping toxicities including fatigue, limb edema, anorexia, rash, mucositis, as well as lymphopenia, thrombocytopenia and transaminase elevation were seen. As a result, RP2D could not be identified, and the study was terminated. No further dose de-escalation is planned.

Sparse PK sampling of trebananib during cycle 2 showed similar drug levels as those observed from prior studies for the $10 \mathrm{mg} / \mathrm{kg}$ dose and PK simulations for $15 \mathrm{mg} / \mathrm{kg}$ [12]. Plasma concentrations of temsirolimus were not assessed in our study, making it difficult to exclude a drug-drug interaction between temsirolimus and trebananib as a cause for the toxicities observed. Although trebananib monotherapy has a favorable toxicity profile and the addition of trebananib to cytotoxic chemotherapy was well tolerated [13], combinations with other antiangiogenic drugs have proven to be much more challenging. The AEs observed with the trebananib and bevacizumab combination included fatigue, diarrhea, nausea and epistaxis, whereas those of combining with motesanib were hypertension, diarrhea, fatigue and gastrointestinal upset [20]. Trebananib plus sorafenib led to diarrhea, palmar-plantar erythrodysesthesia syndrome, alopecia, hypertension and fatigue $[21,22]$. The trebananib and sunitinib combination showed common AEs of diarrhea, fatigue, and hypertension [21]. The patterns of toxicities in our study were comparable to those of other antiangiogenic combination partners tested with trebananib.

The toxicities that we observed were independent of dose level, and greatly exceeded the severity and frequency reported for each agent as monotherapy [23]. These toxicities mirror the experience of combining temsirolimus with other antiangiogenic agents, suggesting the possibility of a pharmacodynamic interaction between temsirolimus and trebananib that leads to excessive toxicity. Temsirolimus in combination with bevacizumab in hepatocellular carcinoma demonstrated significant toxicities including cytopenia, fatigue, mucositis and diarrhea [24]. Likewise, this combination in advanced RCC was associated with a $43 \%$ rate of treatment withdrawal unrelated to disease progression and $36 \%$ incidence of G3 or G4 toxicities [25]. Temsirolimus has also been combined with oral tyrosine kinase inhibitors with anti-angiogenic effects. The combinations of temsirolimus with pazopanib were intolerable due to significant fatigue, leukopenia and electrolyte disturbances [26]. In the phase 1 study of temsirolimus with sunitinib, DLTs observed included rash, thrombocytopenia, stomatitis, diarrhea and stomatitis, and the combination was also found to be intolerable [27]. Based on these experiences, any further studies of temsirolimus with anti-angiogenic targeted drugs should be pursued with great caution.

There was one partial response in a breast cancer patient with PIK3CA mutation. Although the addition of the oral mTOR inhibitor everolimus to endocrine therapy improves progression-free survival in breast cancer [28], temsirolimus showed limited activity [29]. The exact mechanism for this patient's exceptional response to the study drugs is unclear. Targeted mutational profiling was only performed on a subset of patients, limiting our ability to define predictive response biomarkers.

This study explored CAFs and their relationship with treatment. Although there appeared to be a trend of sVCAM-1 level elevation upon dosing, it was not statistically significant. Analysis of CAFs did not appear to be particularly informative. This highlights the challenges of interpretation of CAF data in anti-angiogenic trials, due to the lack of biomarker standardization, pathway redundancies, and variability of treatment administration and $\mathrm{CAF}$ assessment.

We investigated the role of circulating TEMs in predicting clinical efficacy. While measurement of Tie2 and TP in monocytes by flow cytometry was technically feasible, we did not identify discrete populations of Tie2+ and Tie2-monocytes, and instead used a relative measure of surface expression. Our exploratory analyses, which are limited by the low number of evaluable patients with TEMs data (only patients at Princess Margaret Cancer Centre had these assays performed because of requirement for rapid sample processing), found no association between these measurements and response to study treatment. Because mTOR inhibition may have direct effects on monocytes, it is possible that these assays could have greater utility in the setting of a Tie2/ANG directed monotherapy.

In conclusion, the combination of trebananib and temsirolimus was poorly tolerated. Toxicities did not appear to be dose-related suggesting there might be pharmacodynamic interaction between the two drugs. Other approaches to circumvent resistance to anti-angiogenic agents are needed, although potentiation of toxicities may limit the tolerability of anti-angiogenic drug combinations. 
Acknowledgments This research was supported by the National Cancer Institute Grants \#U01-CA-132123 and \#UM1CA186644.

\section{Compliance with ethical standards}

Conflict of Interest The authors declare no conflict of interest for this manuscript.

Ethical approval All procedures performed in studies involving human participants were in accordance with the ethical standards of the institutional and/or nationl research committee and with the 1964 Helsinki declaration and its later amendments or comparable ethical standards.

Open Access This article is distributed under the terms of the Creative Commons Attribution 4.0 International License (http:// creativecommons.org/licenses/by/4.0/), which permits unrestricted use, distribution, and reproduction in any medium, provided you give appropriate credit to the original author(s) and the source, provide a link to the Creative Commons license, and indicate if changes were made.

\section{References}

1. Bergers G, Hanahan D (2008) Modes of resistance to antiangiogenic therapy. Nat Rev Cancer 8:592-603

2. Huang H, Bhat A, Woodnutt G, et al. (2010) Targeting the angpttie2 pathway in malignancy. Nat Rev Cancer 10:575-585

3. Ahmad SA, Liu W, Jung YD, et al. (2001) The effects of angiopoietin- 1 and -2 on tumor growth and angiogenesis in human colon cancer. Cancer Res 61:1255-1259

4. Nambu H, Nambu R, Oshima Y, et al. (2004) Angiopoietin 1 inhibits ocular neovascularization and breakdown of the blood-retinal barrier. Gene Ther 11:865-873

5. Shim WS, Teh M, Bapna A, et al. (2002) Angiopoietin 1 promotes tumor angiogenesis and tumor vessel plasticity of human cervical cancer in mice. Exp Cell Res 279:299-309

6. Thurston G, Wang Q, Baffert F, et al. (2005) Angiopoietin 1 causes vessel enlargement, without angiogenic sprouting, during a critical developmental period. Development 132:3317-3326

7. Oliner J, Min H, Leal J, et al. (2004) Suppression of angiogenesis and tumor growth by selective inhibition of angiopoietin-2. Cancer Cell 6:507-516

8. Bunone G, Vigneri P, Mariani L, et al. (1999) Expression of angiogenesis stimulators and inhibitors in human thyroid tumors and correlation with clinical pathological features. Am J Pathol 155: 1967-1976

9. Etoh T, Inoue H, Tanaka S, et al. (2001) Angiopoietin-2 is related to tumor angiogenesis in gastric carcinoma: possible in vivo regulation via induction of proteases. Cancer Res 61:2145-2153

10. Sfiligoi C, de Luca A, Cascone I, et al. (2003) Angiopoietin-2 expression in breast cancer correlates with lymph node invasion and short survival. Int J Cancer 103:466-474

11. Valenzuela DM, Griffiths JA, Rojas J, et al. (1999) Angiopoietins 3 and 4: diverging gene counterparts in mice and humans. Proc Natl Acad Sci U S A 96:1904-1909

12. Herbst RS, Hong D, Chap L, et al. (2009) Safety, pharmacokinetics, and antitumor activity of amg 386, a selective angiopoietin inhibitor, in adult patients with advanced solid tumors. J Clin Oncol Off J Am Soc Clin Oncol 27:3557-3565
13. Mita AC, Takimoto CH, Mita M, et al. (2010) Phase 1 study of amg 386, a selective angiopoietin 1/2-neutralizing peptibody, in combination with chemotherapy in adults with advanced solid tumors. Clinical Cancer Research: an Official Journal of the American Association for Cancer Research 16:3044-3056

14. Vivanco I, Sawyers CL (2002) The phosphatidylinositol 3-kinase akt pathway in human cancer. Nat Rev Cancer 2:489-501

15. Jones N, Master Z, Jones J, et al. (1999) Identification of tek/tie2 binding partners. Binding to a multifunctional docking site mediates cell survival and migration. J Biol Chem 274:30896-30905

16. Hudes G, Carducci M, Tomczak P, et al. (2007) Temsirolimus, interferon alfa, or both for advanced renal-cell carcinoma. N Engl J Med 356:2271-2281

17. Hess G, Herbrecht R, Romaguera J, et al. (2009) Phase iii study to evaluate temsirolimus compared with investigator's choice therapy for the treatment of relapsed or refractory mantle cell lymphoma. J Clin Oncol Off J Am Soc Clin Oncol 27:3822-3829

18. Eisenhauer EA, Therasse P, Bogaerts J, et al. (2009) New response evaluation criteria in solid tumours: revised recist guideline (version 1.1). Eur J Cancer 45:228-247

19. Cescon DW, Bedard PL, Chow S, et al (2013) Tie2-expressing monocytes as potential biomarkers of angiopoietin-tie2 directed therapies: Correlative analysis of a phase i study of amg386 + temsirolimus. AACR 104th Annual Meeting 2013; Apr 6-10; Washington, DC (Abstract 4647)

20. Hong DS, Kurzrock R, Mulay M, et al. (2014) A phase 1b, openlabel study of trebananib plus bevacizumab or motesanib in patients with solid tumours. Oncotarget 5:11154-11167

21. Hong DS, Gordon MS, Samlowski WE, et al. (2014) A phase i, open-label study of trebananib combined with sorafenib or sunitinib in patients with advanced renal cell carcinoma. Clin Genitourin cancer 12:167-177 e162

22. Rini B, Szczylik C, Tannir NM, et al. (2012) Amg 386 in combination with sorafenib in patients with metastatic clear cell carcinoma of the kidney: a randomized, double-blind, placebo-controlled, phase 2 study. Cancer 118:6152-6161

23. Raymond E, Alexandre J, Faivre S, et al. (2004) Safety and pharmacokinetics of escalated doses of weekly intravenous infusion of cci-779, a novel mtor inhibitor, in patients with cancer. J Clin Oncol Off J Am Soc Clin Oncol 22:2336-2347

24. Knox JJ, Qin R, Strosberg JR, et al. (2015) A phase ii trial of bevacizumab plus temsirolimus in patients with advanced hepatocellular carcinoma. Investig New Drugs 33:241-246

25. Escudier BJ, Negrier S, Gravis G, et al. (2010) Can the combination of temsirolimus and bevacizumab improve the treatment of metastatic renal cell carcinoma (mrcc)? Results of the randomized torava phase ii trial. J Clin Oncol 28:15s, (suppl; abstr 4516)

26. Semrad TJ, Eddings C, Dutia MP, et al. (2013) Phase i study of the combination of temsirolimus and pazopanib in advanced solid tumors. Anti-Cancer Drugs 24:636-640

27. Patel PH, Senico PL, Curiel RE, et al. (2009) Phase i study combining treatment with temsirolimus and sunitinib malate in patients with advanced renal cell carcinoma. Clin Genitourin Cancer 7:24 27

28. Baselga J, Campone M, Piccart M, et al. (2012) Everolimus in postmenopausal hormone-receptor-positive advanced breast cancer. N Engl J Med 366:520-529

29. Fleming GF, Ma CX, Huo D, et al. (2012) Phase ii trial of temsirolimus in patients with metastatic breast cancer. Breast Cancer Res Treat 136:355-363 